J. Clin. Chem. Clin. Biochem.

Vol. 20, 1982, pp. 723-729

\title{
Eine empfindliche Methode zur Bestimmung von Glykosaminoglykanverteilungsmustern, angewendet auf 9 Bandscheiben einer menschlichen Wirbelsäule
}

\author{
Von E. Gurr, R. Schubert, A. Delbrück
}

Institut für Klinische Chemie II, Medizinische Hochschule Hannover und

W. Köller

Abteilung für Krankheiten der Bewegungsorgane und des Stoffwechsels, Medizinische Hochschule Hannover

(Eingegangen am 8. Februar/19. Mai 1982)

Zusammenfassung: Es wird eine Methode zur Bestimmung des Glykosaminoglykanverteilungsmusters in einem isolierten Glykosaminoglykanpool beschrieben, die auf der aufeinanderfolgenden Anwendung der spezifischen Enzyme Hyaluronatlyase, Chondroitinsulfatlyase AC und ABC beruht. Die Bestimmung der Komponenten erfolgt empfindlich über die Metabolitkonzentrationen mithilfe der Hochleistungsflüssigkeitschromatographie (Nachweisgrenzen unter 0,1 nmol). Unverdautes Heparan- und Keratansulfat kann dünnschichtchromatographisch getrennt und mit Hilfe der Uronsäure- bzw. Hexosaminbestimmung quantifiziert werden.

Die mit dieser Methode ermittelten Verteilungsmuster stimmen nur dann mit denen überein, die man mit Hilfe der Ionenaustauschchromatographie (Dowex $1 \times 2$ ) erhält, wenn die Fraktionen enzymatisch charakterisiert werden. Die Anwendung der Methode auf 9 Bandscheiben aus einer menschlichen Wirbelsäule (Anulus fibrosus und Nucleus pulposus jeweils getrennt) ergab

1) keine systematische Veränderung des Verteilungsmusters in Abhängigkeit von der Lage der Zwischenwirbelscheiben im mittleren Abschnitt der Wirbelsäule und

2) im Anulus ein im Vergleich zum Nucleus niedrigeres Verhältnis von Galaktosaminoglykan zu Keratansulfat.

\section{A sensitive method for the analysis of the glycosaminoglycan distribution pattern, applied to 9 intervertebral discs of one human spine}

Summary: A method has been established for analysing the glycosaminoglycan distribution pattern in small specimens from intervertebral discs and other connective tissue materials. The procedure is based on the consecutive digestion by the specific enzymes hyaluronate lyase, chondroitin sulfate lyase $\mathrm{AC}$ and $\mathrm{ABC}$. The quantitative determination of the resulting disaccharides was achieved by high performance liquid chromatography with a detection limit of less than $0.1 \mathrm{nmol}$. The undigested heparan- and keratansulfate were separated from each other by thin layer chromatography and quantified by the colorimetric determination of uronic acid and hexosamine, respectively. Correlation of distribution patterns obtained by this method with those obtained by ion exchange chromatography (Dowex $1 \times 2$ ) is not possible without the additional enzymatic characterization of the ion exchange chromatographic fractions. The method was applied to 9 discs of one human spine (anulus fibrosus and nucleus pulposus separately) with the following results:

1) there were no systematic changes to be seen in the distribution patterns with respect to the location of the discs in the spine, and

2) the galactosaminoglycan/keratansulfate ratio was higher in the anulus fibrosus than in the nucleus pulposus.

\section{Einführung}

Die Glykosaminoglykanẹ sind als Bestandteile der extrazellulären Matrix von weseritlicher Bedeutung für die Struktur und Funktion der Bindegewebe. Die einzelnen
Vertreter dieser Stoffklasse (Chondroitin-4- und -6-sulfat, Heparansulfat, Dermatansulfat, Keratansulfat und Hyaluronat) unterscheiden sich unter anderem in ihrem physikalisch-chemischen Verhalten. $\mathrm{Zu}$ ihrer quantitativen 
Bestimmung werden daher weitgehend Trennverfahren angewandt, die diese Eigenschaften ausnutzen (1-7), jedoch wird die Schärfe der Trennung der einzelnen Komponenten voneinander sowohl durch deren unterschiedliche Kettenlänge und Substitutionsgrad (8) als auch durch die Existenz von Hybridmolekülen beeinträchtigt, wie sie insbesondere von den Chondroitinsulfaten und Dermatansulfat bekannt sind (9-11). Bessere Ergebnisse werden durch eine zusätzliche Charakterisierung der Glykosaminoglykankomponenten durch den Abbau mit Hilfe spezifischer Enzyme erzielt. Dies gilt auch für den Einsatz der Elektrophorese, die als die Methode mit dem geringsten Probebedarf gilt (12-14).

Die Schwierigkeit bei der Anwendung von Trennverfahren zur Differenzierung von Glykosaminoglykanen lassen sich mit einem Analysengang umgehen, bei dem die Spezifität dieser Glykosaminoglykan-abbauenden Enzyme Hyaluronidase (aus Streptomyces hyaluroniticus) (15) und Chondroitinase AC sowie ABC (16) direkt genutzt wird. Mithilfe der Hochleistungsflüssigkeitschromatographie können Hyaluronat, die Chondroitinsulfate 0,4 und 6 sowie Dermatansulfat über ihre Metabolite schnell und exakt quantifiziert werden $(17,18)$. Das verbleibende Keratansulfat und Heparansulfat kann dünnschichtchromatographisch getrennt und charakterisiert werden (19). Das Ziel der vorliegenden Arbeit ist die Ausarbeitung einer Methode zur quantitativen Bestimmung der Glykosaminoglykankomponenten im Mikromaßstab nach dem genannten Prinzip und ihre Anwendung an kleinsten Gewebsproben aus Nucleus pulposus und Anulus fibrosus humaner $Z$ wischenwirbelscheiben.

\section{Material und Methoden}

\section{Reagenzien}

Pronase E, $70000 \mathrm{U} / \mathrm{mg}^{1}$ ), Papain-Kristallsuspension ${ }^{1}$ ); Chondroitinsulfatlyase $\mathrm{AC}$ (EC 4.2.2.5) und Chondroitinsulfatlyase ABC (EC 4.2.2.4) $)^{2}$; Hyaluronatlyase (EC 4.2.99.1) ${ }^{3}$ ); 2-Acetamido-2-desoxy-3-O-( $\beta$ - $D$-gluco-4-enpyranosyluronsäure)- $D$ galactose, 2-Acetamido-2-desoxy-3-O-( $\beta$ - $D$-gluco-4-enpyranosyluronsäure)-4-sulfo- $D$-galactose, 2-Acetamido-2-desoxy-3-O( $\beta$-D-gluco-4-enpyranosyluronsäure)-6-sulfo- $D$-galactose ${ }^{4}$; Dowex $1 \times 2\left(200-400\right.$ mesh) $\left.{ }^{5}\right)$; Dünnschichtfertigplatten (Cellulose, $20 \times 20 \mathrm{~cm})^{1}$ ); HPLC-Fertigsäulen (Shandon APS, $5 \times 100 \mathrm{~mm}, 5 \mu$ Partikel $^{6}$ ), und Hibar LiChrosorb $\mathrm{NH}_{2}$, $4 \times 250 \mathrm{~mm}, 10 \mu$ Partikel) $^{1}$ ); Hyaluronsäure (Nabelschnur) ${ }^{5}$ ); Chondroitinsulfat (Haiknorpel) ${ }^{2}$ ). Dermatansulfat wurde aus Rinderaorten (20), Keratansulfat aus der Cornea von Rinderaugen isoliert (21). Alle übrigen Chemikalien ${ }^{1}$ ) hatten den Reinheitsgrad p.a.

\section{Untersuchungsmaterial}

9 Zwischenwirbelscheiben von thorakal $9 / 10$ bis lumbal $5 /$ sakral 1 eines 39-jährigen Mannes (Todesursache: Luftembolie nach Stichverletzungen) wurden untersucht. Die Bandscheibenentnahme ${ }^{7}$ ) erfolgte $24 \mathrm{~h}$ nach Eintritt des Todes, anschließend

\footnotetext{
1) E. Merck, Darmstadt

3) Calbiochem, Gießen

5) Serva, Heidelberg 6) Gynkotek, Münnchen
7) Herrn Prof. Dr. Löblich, Nordstadtkrankenhaus

2) Sigma Chemie, München

$\left.{ }^{4}\right)$ Miles Biochemicals, Frankfurt danken wir für das Untersuchungsmaterial.
}

wurden die Proben präpariert und danach bei $-28^{\circ} \mathrm{C}$ gelagert. Nach dem Auftauen bei Raumtemperatur wurde zunächst an allen Präparaten ein mechanischer Belastungsversuch durchgeführt (Dauer: $5 \mathrm{~min}$ ); nach Abschluß dieser Versuche wurden die Präparate horizontal aufgeschnitten, Material des zentralen Nucleus und des peripheren ventralen und lateralen Anulus entnommen, diese Proben gefriergetrocknet und danach bis zur biochemischen Analyse wiederum bei $-28^{\circ} \mathrm{C}$ verwahrt. Aus technischen Gründen konnten leider nur 7 Proben der Anuli fibrosi untersucht werden.

\section{Isolierung der Glykosaminoglykane}

Homogenisierung des Gewebes mit anschließender Proteolyse durch Pronase/Papain. Fällung der Proteine mit $\mathrm{HCl}$ bei $\mathrm{pH} 1,3$. Fällung der Glykosaminoglykane aus dem. Überstand nach Dialyse gegen bidest. Wasser mit dem 5-fachen Volumen Natriumacetat-gesättigtem Ethanol. Chromatographie an Sephadex G 25 (fein) mit bidest. Wasser. Bestimmung der Glykosaminoglykane im Ausschlußvolumeñ mit Hilfe der Üronsäure bestimmung (22), Gefriertrocknung.

\section{Bestimmung des Verteilungsmusteris}

Ein Aliquot jeder Probe $\left(1-2 \mu \mathrm{mol}\right.$ Uronsäure in $\left.500 \mu 1 \mathrm{H}_{2} \mathrm{O}\right)$ wurden nacheinander (s. u.) mit Hyaluronatlyase, Chondroitinsulfatlyase $\mathrm{AC}$ und $\mathrm{ABC}$ verdaut (Abb. 1). Abbäüprodukte und

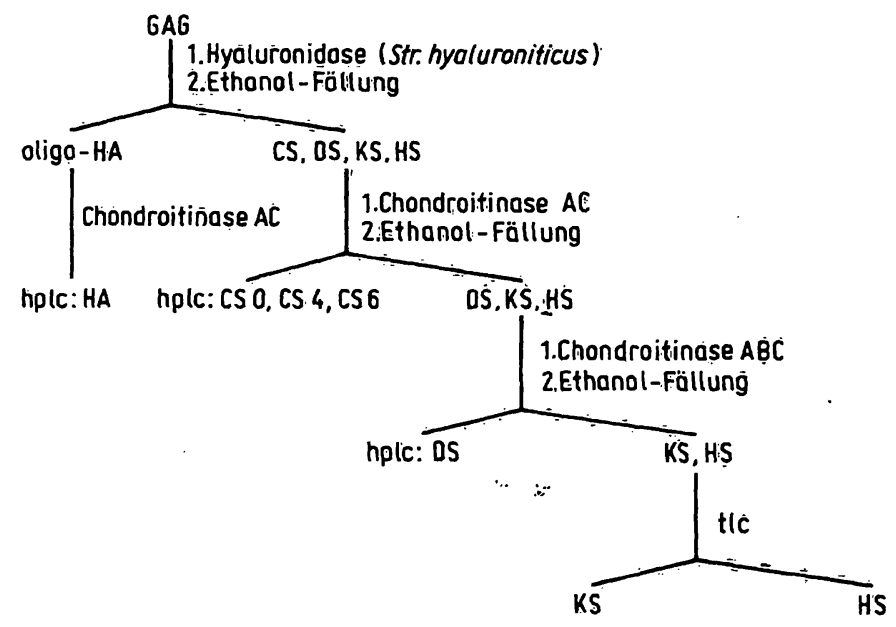

Abb. 1. Das Analysenschema. GAG: Glykosaminoglykan, HA: Hyaluronsäure, CS: Chondroitinsulfat, DS: Dermatansulfat, KS: Keratansulfat, HS: Heparansulfat. hplc: Hochleistungsflüssigkeitschromatographię, tlc: Dünnschichtchromatographie.

Glykosaminoglykañe wurden nach jedem enzymatischen Abbauschritt durch Ethanolfällung voneinander getrennt. Die nichtabgebauten Glykosaminoglykane Heparansulfat und Keratansulfat wurden nach präparativer Dünnschich tchrómatogrạphie an Celluloseplatten durch Elution mit bidest. Wasser isoliert (19). Die Quantifizierung des Heparansulfates erfolgte durch die Ùronsäurebestimmung (22). Keratansulfat wurde als Hexosamin nach Elson-Morgan (23) ohne weitere Differenzierung in Glucosamin und Galaktosamin bestimmt. Die Bestimmung der Disaccharide 2-Acetamido-2-deso xy $\div 3=0 \div(\dot{\beta}-D$-gluco-4-enpyranosyluronsäure )-D-galactose, 2-Acetamido-2-dexoxy-3-O-( $\beta=D$ gluco-4-enpyranosyluronsäure)-4-sulfo-D-galactose und 2-Acet- amido-2-desoxy-3-O-( $\beta-D$-gluco-4-enpyranosyluronsäure) $)$-6sulfo- $D$-galactose in den äthanolischen Ửber̈ständen erfolgte hochleistungsflüssigkeitschromatographisch nach Trocknen im Rotationsverdampfer und Aufnehmen mit 200-400 $\mu 1$ Elutionspuffer (s.u.). Bei einigen Proben erfolgte parallel zu diesem Analysengang die Bestimmung des Glykosaminoglykanverteilungsmusters durčh Chromatographie an Dowex $1 \times 2$ (s.u.). 


\section{Verdaung mit Hyaluronatlyase}

$\mathrm{Zu} 500 \mu \mathrm{l}$ einer wäßrigen Glykosaminoglykanlösung wurden $60 \mu l 0,2 \mathrm{~mol} / 1$ Natriumacetatpuffer ( $\mathrm{pH} 5,2$ ) gegeben und $3 \mathrm{~h}$ bei $60^{\circ} \mathrm{C}$ im Schüttelwasserbad mit $20 \mu 1$ Hyaluronatlyaselösung inkubiert (100 trübungsreduzierende Einheiten in $1 \mathrm{ml} 0,02 \mathrm{~mol} / 1$ Natriumacetatpuffer, $\mathrm{pH} \mathrm{5,2).} \mathrm{Anschließend}$ wurde mit $10 \mu l$ Enzymlösung 2,5 h nachinkubiert, $1 \mathrm{~min}$ im siedenden Wasserbad belassen und mit $3 \mathrm{ml}$ Ethanol $(960 \mathrm{ml} / \mathrm{l})$ über Nacht bei $4^{\circ} \mathrm{C}$ gefällt.

\section{Verdauung mit Chondroitinsulfatlyase $A C$}

$\mathrm{Zu} 500 \mu l$ der wäßrigen Glykosaminoglykanlösung wurden $200 \mu 10,1 \mathrm{~mol} / 1$ Trispuffer, $\mathrm{pH} 8,0$, gegeben und $3 \mathrm{~h}$ bei $37^{\circ} \mathrm{C}$ mit $30 \mu$ l Chondroitinsulfatlyase AC-Lösung inkubiert ( $10 \mathrm{kU} / 1$ bidest. Wasser). Es wurde anschließend $2,5 \mathrm{~h}$ mit $20 \mu \mathrm{l}$ Enzymlösung nachinkubiert, $1 \mathrm{~min}$ im siedenden Wasser bad belassen und mit 3,8 $\mathrm{ml}$ Ethanol $(960 \mathrm{ml} / \mathrm{l})$ über Nacht bei $4{ }^{\circ} \mathrm{C}$ gefällt.

\section{Verdauung mit Chondroitinsulfatlyase $A B C$}

Die Verdauung erfolgte analog der mit Chondroitinsulfatlyase AC mit dem Unterschied, daß die Enzymlösung $10 \mathrm{kU} / 1$ Chondroitinsulfatlyase $\mathbf{A B C}$ in bidest. Wasser enthielt.

\section{Hochleistungsflüssigkeitschromatographie}

Es wurde mit einem Hochleistungsflüssigkeitschromatographen HP 1084 mit Durchflußspektralphotometer und Autosampler gearbeitet ${ }^{8}$ ). Die Detektion erfolgte bei $230 \mathrm{~nm}$. Stationäre Phasen: Shandon APS $(5 \mu \mathrm{m}$ Partikel in $5 \times 100 \mathrm{~mm}$ ShandonFertigsäulen) bzw. LiChrọsorb $\mathrm{NH}_{2}(10 \mu \mathrm{m}$ Partikel in $4 \times 250$ $\mathrm{mm}$ Fertigsäulen). Elutionsmittel: $0,2 \mathrm{~mol} / 1 \mathrm{Natriumace}$ tatpuffer, pH 5,2, dem mit zunehmendem Säulenalter Methanol von $50-500 \mathrm{ml} / 1$ zugesetzt wurde. Die quantitative Auswertung der Chromatogramme erfolgte mithilfe eines externen Standards der entsprechenden Reinsubstanzeen 2-Acetamido-2-desoxy-3-0( $\beta$ - $D$-gluco-4-enpyranosyluronsäure)- $D$-galactose, 2-Acetamido2-desoxy-3=0-( $\vec{\beta}-D$-gluco-4-enpyranosyluronsäure $)$-4-sulfo- $D$ galactose und 2-Acetamido-2-desoxy-3-O-( $\beta-D$-gluco-4-enpyranosyluronsäure)-6-sulfo-D-galactose über die Peakflächen. Jeweils nach 5 Proben wurde das Referenzgemisch chromatographiert. Alle Chromatographien wurden als Doppelbestimmungen durchgeführt. Das Injektionsvolumen betrug $5 \mu$ l.

\section{Chromatographie an Dowex $1 \times 2$}

In einer temperiertèn Säule $\left(1 \times 30 \mathrm{~cm}, 26^{\circ} \mathrm{C}\right)$ wurden etwa 30 nmol Glykosaminoglykane mit einem NaCl:Gradienten eluiert $(30 \mathrm{ml} / \mathrm{h}, 75 \mathrm{ml} / \mathrm{Stufe})$. Den Gradientenstufen wurden die folgenden Glykosaminoglykan-Komponenten zugeordnet $(5,24)$ :

Hyaluronsäure $0,5 \mathrm{~mol} / 1$,

Heparansulfat $1,25 \mathrm{~mol} / 1$

Chondroitinsulfat $1,75 \mathrm{~mol} / 1$,

Dermatansulfat $2,0 \mathrm{mmol} / \mathrm{l}$ und

Keratañsulfat 3,0 mol/1.

Die Quantifizizierung etfolgte durch Uronsäure-(22) bzw. Hexosaminbestimmung (Keratansulfat) (23). Zur weiteren Differenzierung wurde die $1,25 \mathrm{~mol} / 1 \mathrm{NaCl}$-Fraktion nach Dialysse gegen bidest. Wasser mit Chondroitinsulfatlyase $\mathrm{AC}$ verdaut (siehe oben).

\section{Ergebnisse und Diskussion}

\section{Die Methode}

Hyaluronatlyase baut spezifisch Hyaluronșäure (15), Chondroitinsulfatlyase AC Hyaluronsäure sowie die

\footnotetext{
8) Hewlett Packard, Böblingen
}

Chondroitinsulfate und Chondroitinsulfatlyase $\mathrm{ABC}$ außer Hyaluronsäure und den Chondroitinsulfaten Dermatansulfat ab (16). Die Abbauprodukte können chromatographisch in 2-Acetamido-2-desoxy-3-O-( $\beta-D$ gluco-4-enpyranosyluronsäure)-D-galactose, 2-Acetamido2-desoxy-3-0-( $\beta$ - $D$-gluco-4-enpyranosyluronsäure)-4sulfo-D-galactose und 2-Acetamido-2-desoxy-3-O-( $\beta-D$ gluco-4-enpyranosyluronsäure)-6-sulfo-D-galactose aufgetrennt und photometrisch bei $230 \mathrm{~nm}$ bestimmt werden. Für die enzymatische Analyse eines Glykosaminoglykangemisches über die Metabolite muß daher der Abbau in der Reihenfolge Hyaluronatlyase (Hyaluronsäure), Chondroitinsulfatlyase AC (Chondroitin, Chondroitin-4-sulfat und Chondroitin-6-sulfat) und Chondroitinsulfatlyase ABC (Dermatansulfat) erfolgen. Mit der dünnschichtchromatographischen Trennung der nichtabgebauten Heparan- und Keratansulfate ergibt sich der in Abbildung 1 dargestellte Analysengang.

Hyaluronatlyase baut Hyaluronsäure zu einem Oligomerengemisch mit $\alpha-\beta$-ungesättigten Uronsäuren am nicht-reduzierenden Ende ab, dessen quantitative Analyse aufwertdig ist (25). Der Abbau dieses Gemisches mit Chondroitinsulfatlyase AC zu nichtsulfatierten Disacchariden erfolgt vollständig (26). Die Bestimmung der Hyaluronsäure kann daher über die Disaccharide einfacher und aufgrund des größeren Anteils $\alpha-\beta$ ungesättigter Uronsäuren empfindlicher als über die Oligosaccharide durchgefiihrt werden. Unter den angewandten Chromatographiebedingungen unterscheiden sich diese Disaccharide nicht von 2-Acetamido-2-desoxy3-O-( $\beta$ - $D$-gluco-4-enpyranosyluronsäure)- $D$-galactose, die Zuordnung ist jedoch aufgrund des spezifischen enzymatischen Abbaus mit Hyaluronatlyase eindeutig.

Die hochleistungsflüssigkeitschromatographische Bestimmung der Disaccharide ergab in Übereinstimmung mit Lee \& Tieckelmann (17) Proportionalität zwischen Peakfläche und Dișaccharidkonzentration. Die beiden benutzten stationären $\mathrm{NH}_{2}$-Phasen zeigten mit zunehmender Analysenzahl eine Verschlechterung der Trennleistung, die durch eine Erhöhung des Methanolanteils am Elutionspuffer bis zu einer Gesamtanalysenzahl von etwa 200-300 pro Säule kompensiert werden konnte.

Tab. 1. Die Präzision der Analyse der Disaccharidgemische. (A) 13 Analysen in Serie. (B) 10 Analysen eines Referenzgemisches während einer Gesamtzahl von 60 Chromatographien. Erläuterung im Text.

\begin{tabular}{lll}
\hline & $\begin{array}{l}\text { VK(A) } \\
{[\%]}\end{array}$ & $\begin{array}{l}\text { VK }(B) \\
{[\%]}\end{array}$ \\
\hline $\begin{array}{l}\text { 2-Acetamido-2-desoxy-3-O-( } \beta-D \text {-gluco-4- } \\
\text { enpyranosyluronsäure)-D-galactose }\end{array}$ & 3,4 & 2,7 \\
$\begin{array}{l}\text { 2-Acetamido-2-desoxy-3-O-( }(\beta-D-g l u c o-4- \\
\text { enpyranosyluronsäure)-4-sulfo- } D \text {-galactose }\end{array}$ & 2,4 & 2,6 \\
$\begin{array}{l}\text { 2-Acetamido-2-desoxy-3-O-( }-D \text {-gluco-4- } \\
\text { enpyranosyluronsäure)-6-sulfo- } D \text {-galactose }\end{array}$ & 3,0 & 4,0 \\
\hline
\end{tabular}


Tabelle 1 zeigt die Variationskoeffizienten für die Reproduzierbarkeit einer Analyse in der Serie unter konstanten Bedingungen (A) und für die Reproduzierbarkeit eines Standards im Verlaufe von 60 Chromatographien bei Änderung sowohl der Retentionszeiten als auch des Methanolanteils am Elutionspuffer (B).

Die Methode wurde zunächst an einem Gemisch von Hyaluronsäure, Chondroitinsulfat, Dermatansulfat und Keratansulfat erprobt. Das Gemisch wurde aus reinen Lösungen der 4 Komponenten mit bekannten Uronsäure- bzw. Hexosamingehalten (Keratansulfat) hergestellt. Tabelle 2 zeigt die Wiederfindung der Komponenten im Gemisch. Die Bestimmungen von Hyaluronsäure, Chondroitinsulfat und Dermatansulfat stimmen gut mit denen der Reinsubstanzen überein, obgleich in den Reinsubstanzen die Uronsäuren nach Derivatisierung, im Gemisch die Metabolite dagegen direkt photometrisch quantifiziert wurden. Keratansulfat hat die kleinste Wiederfindung (94\%) bei größtem Variationskoeffizienten $(7,9 \%, N=4)$, jedoch ist auch hier die Genauigkeit noch genügend.

Die Inhomogenität der Glykosaminoglykankomponenten führt bei nativen Gemischen bei Anwendung herkömmlicher Verfahren oft zu unbefriedigenden Auflösungen. Die Trennschärfe der hier angegebenen, auf dem enżymatischen Abbau basierenden Methode wurde daher im

Tab. 2. Analyse eines Standardgemisches. Die Konzentration der Komponenten wurde in den Lösungen der Reinsubstanzen über Uronsäure bzw. Hexosamin (Keratansulfat) bestimmt. Die Wiederfindung wurde in einem Gemisch aus den 4 bekannten Lösungen mithilfe des fraktionierten enzymatischen Abbaus über die Metabolitkonzentrationen sowie das Hexosamin (Keratansulfat) ermittelt $(\mathrm{N}=4)$.

\begin{tabular}{llcc}
\hline & $\begin{array}{l}\text { Glykos- } \\
\text { amino- } \\
\text { glykan } \\
\text { [nmol] }\end{array}$ & $\begin{array}{l}\text { Wieder- } \\
\text { findung }\end{array}$ & VK \\
\hline [\%] & {$[\%]$} \\
\hline Hyaluronat & 375 & 96 & 1,6 \\
Chondroitinsulfat & 393 & 99 & 2,1 \\
Dermatansulfat & 339 & 103 & 2,3 \\
Keratansulfat & 298 & 94 & 7,9 \\
\hline
\end{tabular}

Tab. 3. Vergleich zwischen Ionenaustauschchromatographie und fraktioniertem enzymatischen Abbau (alle Angaben in mol\% der Gesamt-Glykosaminoglykane). Die Glykosaminoglykane wurden aus 2 Gallertkernen $(\mathrm{L}, 3$ und $L 3 / 4)$ und einem Faserring $(L 4 / 5)$ von 3 menschlichen lumbalen $Z$ wischenwirbelscheiben isoliert.

\begin{tabular}{lrrrrrr} 
& \multicolumn{1}{c}{ L 2/3 } & \multicolumn{2}{c}{ L 3/4 } & \multicolumn{2}{c}{ L 4/5 } \\
& Dowex & \multicolumn{2}{c}{ Dowex } & \multicolumn{2}{c}{ Dowex } \\
& & Enzym & & Enzym & Enzym \\
\hline Hyaluronat & 12,8 & 15,7 & 8,2 & 9,8 & 3,0 & 4,0 \\
Chondroitinsulfat & 82,1 & 72,1 & 87,1 & 86,3 & 94,7 & 92,0 \\
Dermatansulfat & 3,8 & 4,6 & 0,3 & 1,3 & 0,7 & 2,0 \\
Keratansulfat & 1,3 & 7,6 & 3,7 & 2,5 & 1,7 & 2,0 \\
\hline
\end{tabular}

Vergleich mit der Ionenaustauschchromatographie (Dowex $1 \times 2$ ) mit Glykosaminoglykangemischen aus 3 menschlichen lumbalen Bandscheiben erprobt (Tab. 3). Alle Ionenaustauschchromatographien zeigten eine Fraktion bei $1,25 \mathrm{~mol} / 1 \mathrm{NaCl}$, die $26-30 \%$ der Gesamtsubstanz umfaßte und nach bisherigen K̇íterien Heparansulfat zugeordnet wurde $(5,24)$. Diese Fraktion erwies sich jedoch als Chondroitinase $: A C$-verdaubar und somit als Chondroitinsulfat. Die durch Ionenaustauschchromatographie ermittelten Chondroitinsulfatkonzentrationen in Tabelle 3 entsprechen daher den Summen der 1,25 und $1,75 \mathrm{~mol} / 1 \mathrm{NaCl}-$ Fraktionen.

Die dünnschichtchromatographische Analyse im Anschluß an den enzymatischen Abbau ergab in Übereinstimmung damit weder in diesen noch in den anderen untersuchten Zwischenwirbelscheiben Heparansulfat. Die Keratansulfatbestimmung konnte daher ohne einé vorhergehende präparative Trennung durchgeführt werden. Die mit beiden Methôden vergleichend ermittelten Verteilungsmuster stimmten für die 3 untersuchten Zwischenwirbelscheiben nach der Charakterisierung der 1,25 $\mathrm{mol} / 1 \mathrm{NaCl}$-Fraktion als Chondroitinsulfat gut überein. Die Berechnung der Wiederfindung der in den enzymatischen Verdauungsgang eingesetzten Uronsäuren àls Summe von Hyaluronsäure und Chondroitinsulfat ergab $91 \%(\mathrm{VK}=4 \%, \mathrm{~N} \doteqdot 3)$.

Die Sensitivität der enzymatischen Methode ist gẹgeben durch die Ëmpfindlichkeit dẹ Einzelbèstimmungen. Die untere Grenże für die Bestimmung der Disaccharide aus den enzymatischen Abbauschritten lag unter den hier angewandten hochleistungșflüssigkeitschromàtographischen Bedingungen bei etwa 0,01 nmol pro Chromatographie für jeden der 3 Đisaccharide. In Übereinstimmung mit der Literatur lag die Nachweisempfindlichkeit für Hexosamin (Keratansulfat) bei $10 \mathrm{nmol}(23,27,28)$, die für Uronsäurê (Heparansulfat) bei $1 \mathrm{nmol}$ (22). Die Quantifizierung der Glykosamino= glykankomponenten nach Fraktionierung durch Ionenaustauschchromatographie oder ähnliche Verfähren. erfolgt ebenfalls über die Bestimmung der Uronsäuren bzw. Hexosamine (1-7). Es ergibt sich damit für Hyaluronsäure, Chondroitinsulfat und Dermatansulfat eine 100-fach größere Empfindlichkeit bei der Bestimmung durch den fraktionierten enzymatischen Abbau mit anschließender hochleistungsflüssigkeitschromato: graphischer Bestimmung der Metabólite. Sie liegt in der Größenordnung der nach Elektrophorese durch Alcianblau-Färbung nachweisbaren Glykosaminoglykankomponenten (14). Zusätżlich erhält man jedoch durch die Chromatographie der Disaccharide den Sulfatierungsgrad und den Sulfatierungsort:

Aufgrund der Sensitivität der Ëinzelbestimmungen ist die für die Analyse notwendige Glykosaminoglykanmenge abhängig vom Keratansulfatanteil als dem Anteil mit der niedrigsten Nachweisempfindlichkeit. Die pro Analyse eingesetzte Menge betrug hỉér standardmäßig 
$1,5 \mu \mathrm{mol}$ (bezogen auf den Uronsäuregehalt), sie kann ohne Einbußen an Genauigkeit bei Glykosaminoglykanen aus Bandscheiben auf $100 \mathrm{nmol}$ verringert werden. Eine wesentliche Verbesserung der Empfindlichkeit wird durch die fluorometrische Bestimmung der Hexosamine möglich, mit der ihr Nachweis noch im pmol-Bereich ausfuhrbar ist (29). Durch eine Differenzierung in Glucosamin und Galaktosamin kann weiterhin die Vollständigkeit des enzymatischen Abbaus kontrolliert werden.

Während der Durchfuihrung dieser Arbeit wurden 2 Methoden zur Ermittlung von Glykosaminoglykanverteilungsmustern publiziert, die ebenfalls auf der aufeinanderfolgenden Anwendung der spezifischen Lyasen beruhen $(14,30)$. In beiden Arbeiten erfolgte die Bestimmung der Verteilungsmuster aber über die Quantifizierung nichtabgebauter Glykosaminoglykane nephelometrisch (30) bzw. densitometrisch nach Anfärben mit Alcianblau (14). Die Trennschärfe wird hierbei durch den Einsatz der spezifischen Enzyme verbessert, es sind jedoch Referenzsubstanzen mit gleichen physikalisch-chemischen Eigenschaften zur genauen Quantifizierung erforderlich, da Molekulargewicht und Sulfatierungsgrad jeweils das Meßsignal beeinflussen $(30,31)$. Aussagen über den Sulfatierungsgrad und den Anteil der 4- und 6-sulfatierten Isomere, wie man sie bei der Analyse der enzymatischen Abbauprodukte erhält, lassen beide Methoden nicht zu.

\section{Das Glykosaminoglykanverteilungsmuster in 9 Zwischen- wirbelscheiben einer Wirbelsäule}

In Anwendung dieses Verfahrens konnten die Glykosaminoglykanverteilungsmuster fựr 9 Zwischenwirbel- scheiben einer Wirbelsäule jeweils für den Nucleus pulposus und den Anulus fibrosus ermittelt werden (siehe Tab. 4). Es wurde neben den Hauptbestandteilen Chondroitinsulfat, Keratansulfat und Hyaluronsäure auch Dermatansulfat in beiden Kompartimenten nachgewiesen. Der chemische Nachweis des Dermatansulfates durch die Bestimmung der Iduronsäure konnte wegen des Fehlens einer genügend empfindlichen Methode nicht durchgeführt werden. Der enzymatische Nachweis des Dermatansulfates wird jedoch durch das Ergebnis der methodischen Vergleichsuntersuchung bestätigt, bei der es ebenfalls in Zwischenwirbelscheiben auch durch lonenaustauschchromatographie in ähnlicher Konzentration bestimmt wurde. Bisher ist das Vorkommen von Dermatansulfat in Bandscheiben noch nicht beschrieben worden. Das Verteilungsmuster deutet in Übereinstimmung mit anderen Untersuchungen auf eine Proteoglykanstruktur wie im hyalinen Knorpel hin (32). Wie auch von anderen Autoren beschrieben, wurde für den Nucleus eine mehrfach höhere Konzentration (bezogen auf das Trockengewicht) der Gesamtglykosaminoglykane und der einzelnen Komponenten als im Anulus bestimmt (33-35).

Für eine theoretische, alle Zwischenwirbelscheiben des untersuchten Wirbelsäulenabschnittes repräsentierende Bandscheibe kann aus den Verteilungsmustern jeweils aller Anuli und Nuclei ein arithmetisch gemitteltes Muster berechnet werden, da sich eine systematische Anderung der Konzentration in Abhängigkeit von der Lokalisation der Bandscheiben in der Wirbelsäule bei keiner der Komponenten andeutet. Die mittleren Konzentrationen der Einzelkomponenten (bezogen auf das Trockengewicht, Abb. 2) sind im Nucleus höher ent-

Tab. 4. Das Glykosaminoglykanverteilungsmuster in den Anuli und Nuclei von 9 Zwischenwirbelscheiben einer humanen Wirbelsäule von thoracal $9 / 10$ bis lumbal 5/sakral 1 . Der Gesamtgehalt ergibt sich als Summe aus Uronsäure- und Keratangehalt (in nmol/ mg Trockengewicht). Die Anteile der einzelnen Komponentên sind in mol\% (auf der Basis der Disaccharidbausteine) ạngegeben. Die Verteilungsmuster wurden durch fraktionierten enzymatischen Abbau ermittelt.

\begin{tabular}{|c|c|c|c|c|c|c|c|c|}
\hline & & $\begin{array}{l}\text { Glykos- } \\
\text { aminino- } \\
\text { glykan } \\
{[\mathrm{nmol} / \mathrm{mg}]}\end{array}$ & $\begin{array}{l}\text { Hyaluj- } \\
\text { ronat } \\
\text { [mol\%] }\end{array}$ & $\begin{array}{l}\text { Chondroitin } \\
{[\mathrm{mol} \%]}\end{array}$ & $\begin{array}{l}\text { Chondroitin- } \\
\text { 4-sulfat } \\
\text { [mol\%] }\end{array}$ & $\begin{array}{l}\text { Chondroitin- } \\
\text { 6-sulfat } \\
\text { [mol\%] }\end{array}$ & $\begin{array}{l}\text { Dermatan- } \\
\text { sulfat } \\
\text { [mol\%] }\end{array}$ & $\begin{array}{l}\text { Keratan- } \\
\text { sulfat } \\
\text { [mol\%] }\end{array}$ \\
\hline Anulus & $\begin{array}{l}\text { Th } 9 / 10 \\
\text { Th } 10 / 11 \\
\text { Th } 11 / 12\end{array}$ & $\begin{array}{l}463,1 \\
282,2 \\
219,3\end{array}$ & $\begin{array}{l}5,3 \\
7,0 \\
2,9\end{array}$ & $\begin{array}{r}11,4 \\
9,0 \\
19,9\end{array}$ & $\begin{array}{l}7,5 \\
6,9 \\
6,0\end{array}$ & $\begin{array}{l}29,9 \\
33,9 \\
35,6\end{array}$ & $\begin{array}{l}6,6 \\
5,0 \\
0,1\end{array}$ & $\begin{array}{l}39,4 \\
38,2 \\
35,6\end{array}$ \\
\hline & $\mathrm{Th} 12 / \mathrm{L} 1$ & $=-$ & - & - & - & - & - & - \\
\hline.. & $\begin{array}{ll}\mathrm{L} & 1 / 2 \\
\mathrm{~L} & 2 / 3 \\
\mathrm{~L} & 3 / 4 \\
\mathrm{~L} & 4 / 5 \\
\mathrm{~L} & 5 / \mathrm{S} 1\end{array}$ & $\begin{array}{l}- \\
202,6 \\
207,7 \\
269,3 \\
317,1\end{array}$ & $\begin{array}{r}6,7 \\
7,2 \\
10,1 \\
11,3 \\
12,4\end{array}$ & $\begin{array}{l}8,5 \\
0 \\
8,5 \\
8,7 \\
8,9\end{array}$ & $\begin{array}{l}5,7 \\
3,7 \\
5,4 \\
6,4 \\
6,8\end{array}$ & $\begin{array}{l}38,1 \\
45,2 \\
30,4 \\
36,1 \\
40,4\end{array}$ & $\begin{array}{l}5,4 \\
3,1 \\
6,9 \\
0,8 \\
3,1\end{array}$ & $\begin{array}{l}35,6 \\
40,8 \\
38,9 \\
36,7 \\
28,4\end{array}$ \\
\hline Nuçleus & 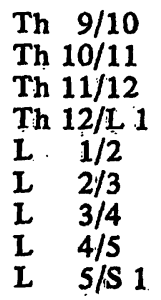 & $\begin{array}{r}730,8 \\
998,0 \\
777,1 \\
739,0 \\
790 / 8 \\
623,9 \\
1021,0 \\
847,7 \\
695,8\end{array}$ & $\begin{array}{r}4,2 \\
9,6 \\
10,0 \\
3,3 \\
4,6 \\
7,3 \\
9,2 \\
10,9 \\
7,3\end{array}$ & $\begin{array}{r}9,4 \\
10,8 \\
9,5 \\
10,8 \\
7,6 \\
10,5 \\
10,1 \\
9,8 \\
7,4\end{array}$ & $\begin{array}{l}6,2 \\
7,1 \\
6,1 \\
7,6 \\
7,0 \\
7,0 \\
7,2 \\
5,9 \\
5,7\end{array}$ & $\begin{array}{l}37,3 \\
35,6 \\
35,9 \\
43,7 \\
44,6 \\
42,3 \\
41,7 \\
38,3 \\
38,6\end{array}$ & $\begin{array}{l}6,2 \\
2,0 \\
3,5 \\
2,3 \\
2,8 \\
2,4 \\
3,0 \\
2,4 \\
5,9\end{array}$ & $\begin{array}{l}36,7 \\
34,9 \\
35,1 \\
32,2 \\
33,4 \\
30,7 \\
28,8 \\
32,8 \\
35,0\end{array}$ \\
\hline
\end{tabular}




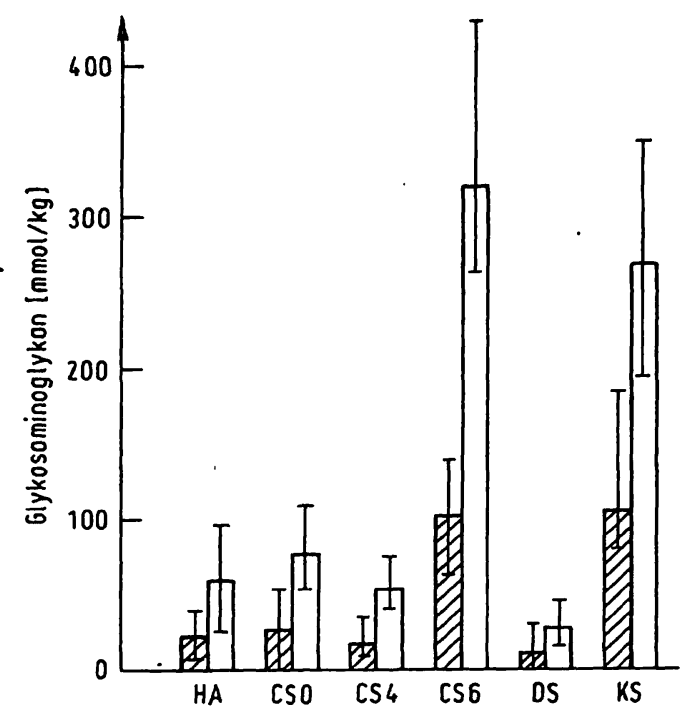

Abb. 2. Das gemittelte Glykosaminoglykanverteilungsmuster des untersuchten Wirbelsäulenabschnittes in Anulus und Nucleus mit Angaben der Extremwerte. Konzentrationen in $\mathrm{nmol} / \mathrm{mg}$ Trockengewicht.

$\square$ : Anulus, $\square$ : Nucleus.

CS: Chondroitinsulfat, DS: Dermatansulfat, KS: Keratansulfat.

sprechend des höheren Gesamtglykosaminoglykangehaltes. Berechnet man jedoch den Anteil der einzelnen Komponenten am Gesamtglykosaminoglykanpool, so erhält man je ein relatives mittleres Verteilungsmuster für Anulus und Nucleus (Abb. 3). Es deuten sich Unterschiede nur zwischen den Chondroitin-6-sulfat- und Keratansulfatanteilen an.

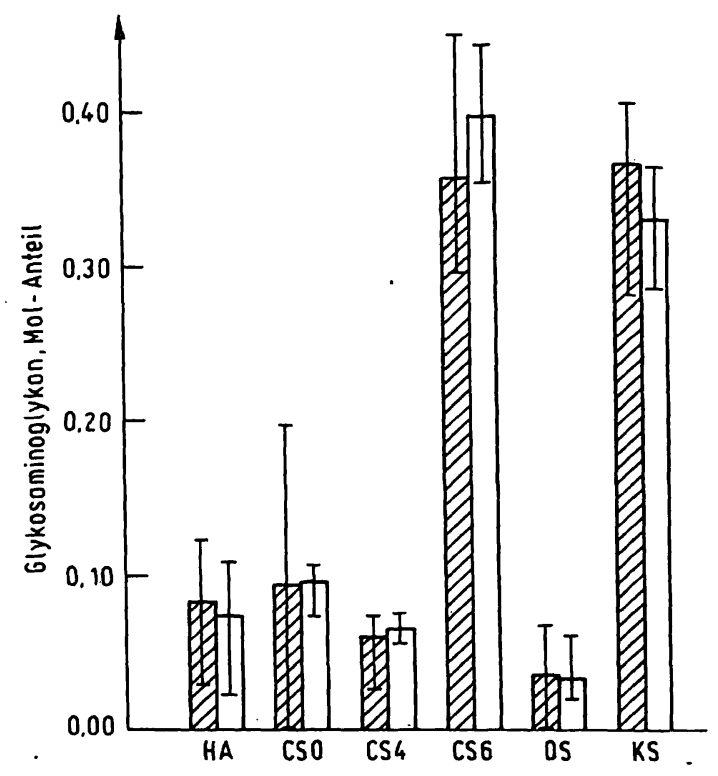

Abb. 3. Das gemittelte relative Verteilungsmuster des untersuchten Wirbelsäulenabschnittes mit Angabe der Extremwerte. Konzentrationsangaben in mol\% des Gesamtglykosaminoglykanpools.

$\square$ : Anulus, $\square$ : Nucleus.

CS: Chondroitinsulfat, DS: Dermatansulfat, KS: Keratansulfat.
Proteoglykane aus Zwischenwirbelscheiben zeigen ähnlich wie die aus hyalinem Knorpel drei verschiedene Strukturelemente: eine vorwiegend chondroitinsulfathaltige Region, eine vorwiegend keratansulfathaltige Region und eine glykosaminoglykanfreie Hyaluronatbindungsregion (32, $34,36-38)$. Ein relatives Maß für die Größe von Proteoglykanen ist der Quotient aus Chondroitinsulfat- und Keratansulfatanteil, der sich z.B. bei degenerativen Veränderungen wegen der Verkürzung der chondroitinsulfathaltigen Region verkleinert (38-40). Mit der Annahme, daß das Dermatansulfat aufgrund seiner Hybridstruktur der chondroitinsulfathaltigen Region zuzuordnen ist (9-11), wurde für diesen Wirbelsäulènäbschnitt der Quotient aus den Galaktosaminoglykanen und Keratansulfat berechnet (Tab. 5). Der mittlere Quotient ist für den Nucleus signifikant höher. Man kann daher auf im Nucleus größere Proteoglykanmolekiile mit höherem Chondroitinsulfatgehalt pro Molekül schließen.

Tab. 5. Der Quotient aus galaktosaminhaltigen Glykosaminoglykanen und Keratansulfat, berechnet aus den relativen molaren Konzentrationen (Tab. 4). Der Unterschied zwischen Anulus und Nucleus ist signifikant ( $t-T e s t$, $p=0,05$ ).

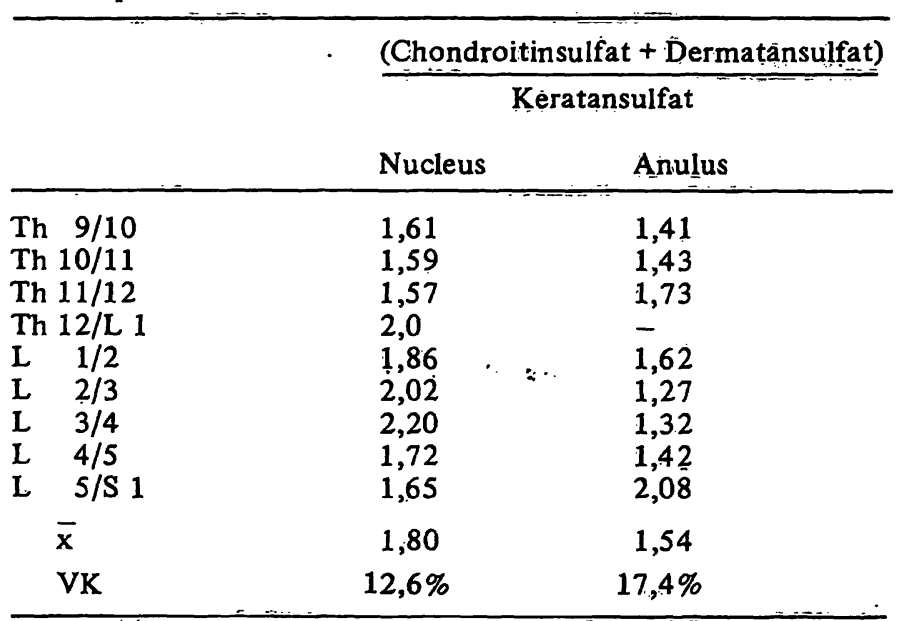

Der Anteil hyaluronatgebundener Proteoglykane beträgt nach Stevens et al. im Anulus 60\% und im Nucleus 25\% (32). Da die relative mittlere Hyaluronatkonzentration in beiden Kompartimenten der Bandscheiben des untersuchten Wirbelsäulenabschnittes gleich ist, ergibt sich für den Nucleus ein kleineres Verhältnis von molarer Proteoglykan- zu Hyaluronatkonzentration. Der niedrigere Komplexbildungsanteil im Nucleus kann daher nicht durch eine im Verglèich zu molaren Proteoglykankonzentration erniedrigte Hyaluronatkonzentration bedingt sein, wie Hardingham et al. vermuten (34); sondern läßt eher auf eine veränderte Hyaluronatbindungsregion und möglicherweise Änderungen in der Konzentration des Link-Proteins schließen. Dieses jedoch muß erst noch durch Strukturuntersuchungen intakter Bandscheibenproteoglykane bestätigt werden. -i 


\section{Danksagung}

Die Arbeit wurde mit Unterstützung der Deutschen Forschungsgemeinschaft (SFB 54) durchgeführt. Sie wurde zum Teil auf

\section{Literatur}

1. Wessler, E. (1968) Anal. Biochem. 26, 439-444.

2. Scott, J. E. (1955) Biochim. Biophys. Acta 18, 428-429.

3. Buddecke, E. (1960) Hoppe-Seyler's Z. Physiol. Chemie $318,33-55$.

4. Meyer, U., Davidson, E., Linker, A. \& Hoffmann, P. (1956) Biochim. Biophys. Acta 21, 506-518.

5. Schiller, S., Slover, G. A. \& Dorfman, A. (1961) J. Biol. Chem. 236, 983-987.

6. Klemer, A. \& Mempel, D. (1965) Z. Naturforsch. 20b, $553-559$.

7. Greiling, H., Herbertz, T. \& Stuhlsatz, H. W. (1964) HoppeSeyler's Z. Physiol. Chem. 336, 149-162.

8. Camper, W. D. \& Laurent, T. C. (1978) Physiol. Rev. 58, $255-315$.

9. Stuhlsatz, H. W. \& Greiling, H. (1976) The Methodology of Connective Tissue Research, S. 129-136, (Hall, D. A., ed.) Joynson-Bruvvers Ltd. Oxford.

10. Fransson, L. A. (1968) J. Biol. Chem. 243, 1504-1510.

11. Fransson, L. A. (1968) Archiv f. Kemi 29, 95-98.

12. Murata, K. \& Bjelle, A. O. (1980) Conn. Tissue Res. 7, 143-156.

13. Capelletti, R., Del Rosso, M. \& Chiarugi, V. (1980) Anal. Biochem. 105, 430-435.

14. Breen, M., Knepper, P. A., Weinstein, H. G., Black, L. J., Lewandowski, D. L. \& Baltrus, B. M. (1981) Anal. Biochem. $113,416-422$.

15. Ohya, T. \& Kaneko, K. (1970) Biochim. Biophys. Acta 198, 607-609.

16. Yamagata, T., Saito, H., Habuchi, O. \& Suzuki, S. (1968) J. Biol. Chem. 243, 1523-1535.

17. Lee, G. J. \& Tieckelmann, H. (1979) Anal Biochem. 94, $231-236$

18. Hjerpe, A., Antonopoulos, C. A. \& Engfeld, B. (1979) J. Chromatogr. 171, 339-344.

19. Humbel, R. \& Chamoles, N. A. (1972) Clin. Chim. Acta 40, 290-293.

20. Stuhlsatz, H. W. \& Greiling, H. (1976) The Methodology of Connective Tissue Research, S. 137-146, (Hall, D. A. ed.) Joynson-Bruvivers Ltd., Oxford. dem Symposium „Arthritis - Models and Mechanisms"vom 24. -25. September 1980 in Hannover vorgetragen. Frl. M. Arlart und Frl. K. Hampel sei für die Hilfe bei der experimentellen Durchführung gedankt.

21. Mathews, M. B. (1976) The Methodology of Connective Tissue Research, S. 147-151, (Hall, D. A. ed.) JoynsonBruvvers Ltd., Oxford.

22. Bitter, A. \& Muir, H. (1962) Anal. Biochem. 4, 330-334.

23. Blumenkranz, N. \& Asboe-Hansen, G. (1976) Clin. Biochem. 9, 269-274.

24. Delbrück, A. (1968) Z. Klin. Chem. Klin. Biochem. 6, 460-466.

25. Shimada, E. \& Matsumura, G. (1980) J. Biochem. 88 , 1015-1023.

26. Gurr, E., unveröffentlichtes Ergebnis.

27. Good, T. A. \& Bessmann, S. P. (1964) Anal. Biochem. 9, 253-262.

28. Oguchi, M. \& Oguchi, M. S. (1979) Anal. Biochem. 98, 433-437.

29. Carroll, S. F. \& Nelson, D. R. (1979) Anal. Biochem. 98, 190-197.

30. Gressner, A. M., Scherer, R., Stuhlsatz, H. W. \& Greiling, H. (1981) J. Clin. Chem. Clin. Biochem. 19, 221-222.

31. Breen, M., Weinstein, H. G., Anderson, M. \& Veis, A. (1970) Anal. Biochem. 35, 146-159.

32. Stevens, R. L., Ewins, R. F. J., Revell, P. A. \& Muir, H. (1979) Biochem. J. 179, 561-572.

33. Urban, J. \& Maroudas, A. (1980) Clinics in Rheum. Diseases $6,51-76$.

34. Hardingham, T. E. \& Adams, P. (1976) Biochem. J. 159, 143-147.

35. Urban, J. \& Maroudas, A. (1979) Biochim. Biophys. Acta $586,166-178$.

36. Wiesländer, J. \& Heinegard, D. (1979) Biochem. J. 179, $35-45$.

37. Swann, D. A., Powell, S. \& Sotman, S. (1979) J. Biol. Chem. 254, 945-954.

38. Roughley, P. J. \& White, R. J. (1980) J. Biol. Chem. 255, 217-224.

39. Inerot, S., Heinegard, D., Audell, L. \& Olssau, S. (1978) Biochem. J. 169, 143-156.

40. Bayliss, M. T. \& Ali, S. J. (1978) Biochem. J. 176, 683-693.

Dr. Eberhard Gurr Krankenhaus Oststadt - Zentrallabor Podbielskistr. 380 D-3000 Hannover 51 


$$
\text { 。 }
$$

